\section{Kounis syndrome: Aspects of incidence and epidemiology}

To the Editor: Kounis syndrome is a hypersensitivity coronary disorder induced by environmental factors, foods, drugs, conditions and stents. It manifests as coronary spasm, acute myocardial infarction and stent thrombosis, and affects the cerebral, mesenteric and coronary arteries. Allergic, hypersensitivity, anaphylactic and anaphylactoid reactions are associated with this syndrome. Kounis syndrome has been observed in every race, age group (2 - 90 years) and geographical location. ${ }^{[1]}$ It is not a rare disease, but it is infrequently reported in clinical practice because of missed, unrecognised and/or undiagnosed cases. Furthermore, there is a paucity of large prospective trials, determining its prevalence and exact incidence. In a very interesting report published in the $S A M{ }^{[2]}$ a 39-year-old man with a previous history of exercise-induced asthma and allergy to cefixime developed type I Kounis syndrome following an allergic reaction to ciprofloxacin. The authors correctly commented on the paucity of data concerning the incidence and prevalence of Kounis syndrome in South Africa and elsewhere. We therefore consider the following aspects on its incidence and epidemiology to be of additional value:

The incidence of anaphylaxis with circulatory symptoms is estimated at 7.9 - 9.6/100 000 inhabitants per year with a case fatality rate of $0.0001 \% .{ }^{[3]}$

In the only retrospective study published so far, the incidence of Kounis syndrome at emergency departments in 1 year among all admissions and allergy patients was 19.4/100 000 (27/138 911) and $3.4 \%(27 / 793)$, respectively. ${ }^{[4]}$

Of the 51 cases of Kounis syndrome reported to the International Pharmacovigilance Agency (VigiBase) in the period 2010 - 2014, almost half (22 reports) were in 2014. Most cases occurred in the USA, and non-steroidal anti-inflammatories were the most frequent trigger drugs. ${ }^{[5]}$

Kounis syndrome prevalence was estimated to be at $0.002 \%$ in the catheterisation laboratory of a tertiary hospital in Istanbul, Turkey. ${ }^{[6]}$

The annual incidence of Kounis syndrome in the district of Achaia, Greece, with a population of 300000 inhabitants, was estimated at 4.33 cases $/ 100000$ inhabitants..$^{[7]}$

Troponin levels were found to be significantly raised in 31 patients admitted to the emergency department and suffering from anaphylaxis, angioedema, urticaria and urticaria-angioedema in comparison with 125 healthy controls. Systematic troponin measurement in patients with acute allergic reactions is advisable in order to detect and treat potential myocardiac injury. ${ }^{[8]}$
Finally, in 300 anaphylaxis cases, myocardial injury was present in $7.3 \%$ of patients. Various cardiomyopathies, including Kounis syndrome and Takotsubo cardiomyopathy, were also observed in patients with myocardial injury. ${ }^{[9]}$

All the above denotes that the incidence of Kounis syndrome is higher that it has been estimated so far. Kounis syndrome has mostly been encountered in southern Europe, especially Turkey, Greece, Italy and Spain. ${ }^{[10]}$ This geographical variation could be attributed to the increased awareness of physicians of the existence of Kounis syndrome, climate and environmental conditions that result in pollen cross-reactivities and hymenoptera exposures, overconsumption of medicines, or inadequacy of preventative measures. It is anticipated that with increased awareness of the existence of Kounis syndrome and the conducting of large prospective trials, the true estimation of its incidence will be achieved.

\section{Nicholas G Kounis}

Department of Medical Sciences, Western Greece Highest Institute of Education and Technology, Patras, Achaia, Greece

ngkounis@otenet.gr

\section{Nicholas Grapsas, Dimitrios Lianas, George D Soufras}

Department of Cardiology and Emergency Medicine, Saint Andrews State General Hospital, Patras, Achaia, Greece

\section{Nicholas Patsouras}

Department of Cardiology, University of Patras Medical School, Patras, Achaia, Greece

1. Kounis NG, Giannopoulos S, Soufras GD, Kounis GN, Goudevenos J. Foods, drugs and environmental factors: Novel Kounis syndrome offenders. Intern Med 2015;54(13):1577-1582. DOI:10.2169/ internalmedicine. 54.3684

2. Ntuli PM, Makambwa E. Kounis syndrome. S Afr Med J 2015;105(10):878. DOI:10.7196/ SAMJnew. 8767

3. Helbling A, Hurni T, Mueller UR, Pichler WJ. Incidence of anaphylaxis with circulatory symptoms: A study over a 3-year period comprising 940000 inhabitants of the Swiss Canton Bern. Clin Exp Allergy 2004;34(2):285-290. DOI:10.1111/j.1365-2222.2004.01882

4. Akoz A, Tanboga HI, Emet M, et al. A prospective study of Kounis syndrome: Clinical experience and cardiac magnetic resonance imaging findings for 21 patients. Acta Med Mediterraea 2013;29:811-816 5. Renda $F$, Landoni $G$, Trotta F, et al. Kounis syndrome: An analysis of spontaneous reports from internationa pharmacovigilance database. Int J Cardiol 2016;203:217-220. DOI:10.1016/jijcard.2015.10.003

6. Biteker M. Current understanding of Kounis syndrome. Exp Rev Clin Immunol 2010;6(5):777-788. DOI:10.1586/eci.10.47

7. Kounis NG, Mazarakis A, Tsigkas G, Giannopoulos S, Goudevenos J. Kounis syndrome: A new twist on an old disease. Future Cardiol 2011;7(6):805-824. DOI:10.2217/fca.11.63

8. Lippi G, Buonocore R, Schirosa F, Cervellin G. Cardiac troponin I is increased in patient admitted to the emergency department with severe allergic reactions. A case-control study. Int J Cardiol 2015;194:68-69. DOI:10.1016/i.ijcard.2015.05.093

9. Cha YS, Kim H, Bang MH, et al. Evaluation of myocardial injury through serum troponin and echocardiography in anaphylaxis. Am J Emerg Med 2016;34(2):140-144. DOI:10.1016/j. ajem.2015.09.038

10. Kounis NG, Soufras GD, Davlouros P, Tsigkas G, Hahalis G. Combined etiology of anaphylactic cardiogenic shock: amiodarone, epinephrine, cardioverter defibrillator, left ventricular assist devices and the Kounis syndrome. Ann Card Anaesth 2015;18(2):261-264. DOI:10.4103/0971-9784.154498

S Afr Med J 2016;106(5):426. DOI:10.7196/SAMJ.2016.v106i5.10680 